\title{
El agua en la literatura educativa de las dos últimas décadas. Una revisión sistemática
}

\author{
Alejandra Ramírez-Segado (iD, María Rodríguez-Serrano (iD, Alicia Benarroch Benarroch (iD) \\ Departamento de Didáctica de las Ciencias Experimentales, Facultad de Ciencias de la Educación y del \\ Deporte de Melilla, Universidad de Granada.España.alermzsgd@ugr.es,mariarodriguez@ugr.es, \\ aliciabb@ugr.es.
}

[Recibido: 1 marzo 2020. Revisado: 15 junio 2020. Aceptado: 6 noviembre 2020]

\begin{abstract}
Resumen: Este artículo de revisión de la literatura educativa sobre el agua recoge información relevante sobre las dificultades de comprensión del alumnado con esta temática así como estrategias educativas aportadas por la comunidad investigadora. Tras una metodología sistematizada, se analizaron 47 artículos, pertenecientes al período 2000-2018. El análisis se estructura en torno a los contenidos curriculares asociados al agua. Los resultados indican que la etapa educativa más investigada es la de primaria, seguida de secundaria obligatoria; las menos investigadas son la etapa de infantil y la universitaria. Asimismo, se concluye que, según el contenido principal investigado, se ha prestado escasa atención al papel que desempeñan los seres vivos, y en concreto, los humanos, en las investigaciones didácticas relacionadas con el agua.
\end{abstract}

Palabras clave: agua; enseñanza; dificultades de aprendizaje; revisión bibliográfica; propuestas curriculares.

Water in educative literature during the last two decades. A systematic review

Abstract: This review article of educative literature about water summarizes relevant information about the comprehension difficulties of students dealing with this topic together with educative strategies given by the research community. After a systematized methodology, 47 articles belonging to the 2000-2018 period were analyzed. This analysis was structured around the curricular contents of water. The results point that primary education is the most researched educational stage, followed by compulsory secondary education; the ones that are the least researched are preschool and university stages. Likewise, one can conclude that, according to the main researched content, not much attention has been paid to the role of living things, and in particular, human beings, in didactic research related to water.

Keywords: water; teaching; learning difficulties; bibliographical review; curricular contents.

Para citar este artículo: Ramírez-Segado, A., Rodríguez-Serrano, M. y Benarroch, A. (2021) El agua en la literatura educativa de las dos últimas décadas. Una revisión sistemática. Revista Eureka sobre Enseñanza y Divulgación de las Ciencias 18(1), 1107. doi: 10.25267/Rev_Eureka_ensen_divulg_cienc.2021.v18.i1.1107

\section{Introducción}

El agua es uno de los contenidos más extendidos en el currículum escolar de todos los niveles educativos, desde infantil hasta bachillerato. A pesar de ello, las percepciones, ideas y concepciones del alumnado sobre el agua no han sido ampliamente estudiadas (HavuNuutinen, Kärkkäinen y Keinonen 2011). Cuando se trata de diseñar actividades sobre el agua, la información didáctica de la que dispone el profesorado acerca de las dificultades de los estudiantes sobre el agua se encuentra muy dispersa y, en muchos casos, sesgada. Si bien esto puede ocurrir con cualquier contenido, en el caso del agua, la dificultad se acentúa, quizás porque abarca una realidad especialmente compleja en la que se entrelazan numerosos factores de diversa índole.

El carácter ubicuo del agua en nuestro planeta y su importancia, específicamente, sobre nuestra salud y condiciones de vida, hacen que el agua sea una de las temáticas más complejas y poliédricas de nuestra existencia. Es un tema en el que se mezclan numerosos factores naturales, culturales, económicos, históricos y emocionales. Afrontar su estudio desde 
cualquier perspectiva, ya sea física, química, económica, emocional, cultural, etc., y, por supuesto, también desde la visión didáctica, tiene siempre limitaciones.

En el currículum escolar ocupa una posición privilegiada por derecho propio como contenido estrella para la educación ambiental y para formar a una ciudadanía alfabetizada ambientalmente, pues solo con una amplia formación en el comportamiento del agua en la naturaleza se puede discernir, opinar y enjuiciar las opiniones vertidas en las redes y medios sociales y contribuir a su resolución. El agua es un bien escaso y poco cuidado, por lo que su contaminación, escasez o reparto, suele ser fuente de disputas constantes entre comunidades o países. No en vano se le ha llamado el nuevo «oro» del planeta. Conseguir la formación ciudadana en estas controversias es fundamental para nuestra supervivencia. A pesar de esta importancia, y aunque pueda ser sorprendente, no hay muchos estudios que recopilen el conocimiento didáctico sobre el agua.

En este trabajo se pretende realizar una revisión bibliográfica sobre la enseñanza y/o aprendizaje del agua y de sus contenidos curriculares. Se trata de contribuir a las siguientes preguntas de investigación:

1. ¿Qué contenidos curriculares relacionados con el agua han sido más y menos investigados?

2. ¿Qué se sabe acerca de las dificultades de aprendizaje y de las estrategias de enseñanza de los contenidos relacionados con el agua?

\section{Antecedentes teóricos}

Algunas revisiones parciales sobre la enseñanza-aprendizaje del agua son: monográfico sobre aguas subterráneas de Enseñanza de las Ciencias de la Tierra, núm. 15 (Calvo, Reyero, Vidal, Morcillo y García 2007; Márquez y Bach 2007; Nebot 2007; Reyero, Calvo, Vidal, García y Morcillo 2007); Hernández (2014); Fernández Arroyo (2012a) y Henriques (2002). No obstante, la mayor parte de los estudios se focalizan en analizar las ideas de los estudiantes acerca de algunos fenómenos particulares y separan los aspectos físicos, químicos y ambientales unos de otros. Las ideas sobre el agua no se suelen considerar sistemáticamente en la escuela, más bien se concentran en algunos aspectos específicos. Sin embargo, los problemas ambientales y científicos actuales, como el cambio climático, la sostenibilidad y la contaminación, están muy vinculados a las cuestiones de los recursos hídricos, su efecto sobre la vida misma, el ciclo de vida y los seres humanos.

Estos estudios revelan que la imagen que tienen los estudiantes del agua y del ciclo del agua es incompleta y, en muchos casos, errónea. Algunos aspectos destacables son:

a) El alumnado no comprende el rol significativo que tiene el agua para la vida. Por ejemplo, no relacionan las cuencas hídricas con su vida cotidiana. Para los escolares, las cuencas hídricas existen en los paisajes naturales, pero no en los paisajes urbanos donde ellos viven y juegan (Shepardson, Harbor y Wee 2005).

b) Hay una escasa percepción de la necesidad de conservar el agua (en cantidad y calidad), frente a otros problemas ambientales, por ejemplo, los relacionados con la degradación del medio (contaminación atmosférica, mareas negras...) (Havu-Nuutinen et al. 2011).

c) La percepción sobre el agua está influenciada por la cobertura de los libros de texto y de los medios de comunicación. Concretamente, el concepto de recurso natural se aplica más a los recursos energéticos que al agua. Asimismo, las representaciones gráficas del ciclo del agua en los manuales escolares prescinden de la influencia humana, como si ello fuera posible, dando una imagen cíclica e inacabable del agua. Abbott et al. (2019) destacan que 
la omisión de los seres vivos y sobre todo, de los humanos, en el estudio del agua puede ser problemática e inducir a la idea de que este recurso no está influenciado por nuestras acciones.

d) En cuanto a los procesos de enseñanza y aprendizaje, se comprueba que las estrategias Ciencia-Tecnología-Sociedad (CTS) son eficaces para alcanzar mejores conocimientos sobre el agua (Havu-Nuutinen, Kärkkäinen y Keinonen 2017). También se ha indagado en las etapas de progresión en los logros de las explicaciones del alumnado a través de la escolaridad. Estas etapas comienzan con la identificación del agua únicamente en los contextos visibles y familiares, y finalizan con la plena conciencia no solo de su ubicación en todos sus estados físicos y en todos los seres vivos e inertes, sino también de las limitaciones tecnológicas que existen para proporcionar agua dulce (Gunckel, Covitt, Salinas y Anderson 2012). En esta última etapa, el estudiante es capaz de comprender que, si no fuera por el coste y por los daños ambientales, el agua dulce se podría extraer indefinidamente del agua del mar y la escasez del agua sería una completa entelequia.

\section{Metodología}

La revisión bibliográfica se ha llevado a cabo en diferentes etapas, esquematizadas en la figura 1, tituladas planificación, búsqueda, selección y análisis.

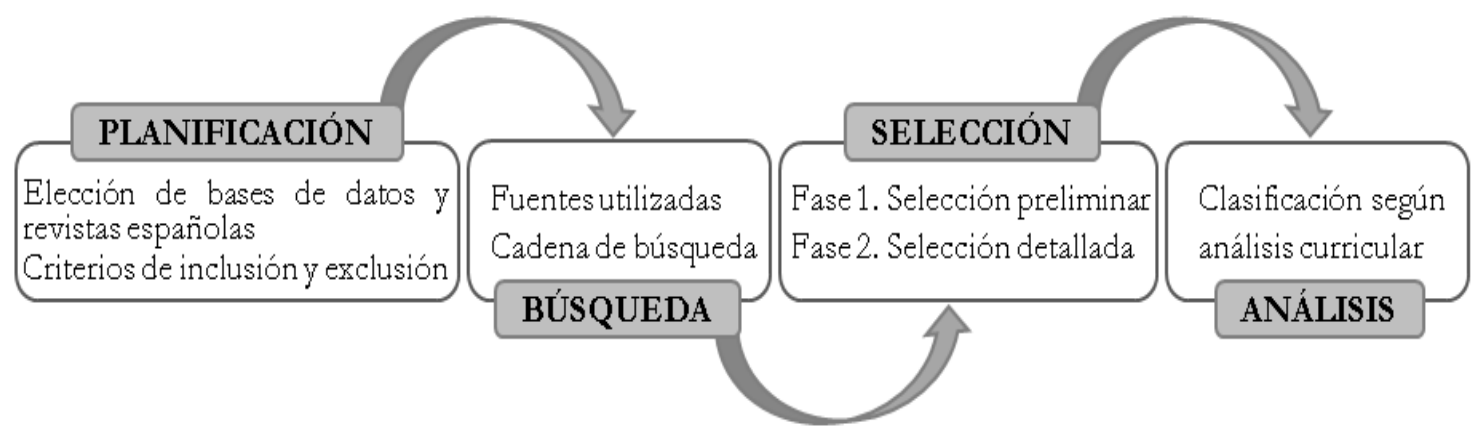

Figura 1. Esquema del método de revisión utilizado

\section{Planificación y búsqueda de artículos}

El proceso se inició a principios de octubre de 2018 con la selección de los criterios de búsqueda específicos para lograr los objetivos planteados. Las bases de datos seleccionadas fueron Web of Science (WOS en adelante), en su colección principal, y Scopus, que son las bases de datos más amplias y reconocidas en el ámbito de las ciencias sociales. También se realizó una búsqueda exhaustiva en las principales revistas españolas en el área de Didáctica de las Ciencias Experimentales, concretamente, en Alambique, Didáctica de las Ciencias Experimentales y Sociales, Enseñanza de las Ciencias, Enseñanza de las Ciencias de la Tierra, Investigación en la Escuela y Revista Eureka sobre Enseñanza y Divulgación de las Ciencias.

$\mathrm{Al}$ introducir las revistas españolas más especializadas en la didáctica de las ciencias, se está generando una asimetría en la muestra de artículos hacia la investigación realizada en España y hacia la inclusión de artículos publicados en revistas no indexadas. No obstante, y a pesar de ello, se tomó esta decisión para cubrir con mayor exhaustividad el campo temático de interés al menos en el contexto más cercano.

El criterio definido para seleccionar los documentos potencialmente relevantes fue una cadena de búsqueda compuesta de palabras clave: (water AND teaching AND learning) y en español: (agua AND enseñanza AND aprendizaje). La búsqueda se especificó para título, resumen y palabras clave. Como fecha de publicación, se limitó la búsqueda al periodo comprendido entre los años 2000 y 2018 (ambos inclusive). Además, otro filtro fue el área de conocimiento: 
Education Educational Research para WOS y Social Sciences para Scopus. La misma cadena de búsqueda fue también utilizada para las revistas españolas que cuentan con buscador. En caso contrario, se realizó una búsqueda de todos los artículos y volúmenes de la revista.

El resultado obtenido al aplicar la cadena de búsqueda en las diferentes fuentes empleadas fue de 1633 artículos (280 trabajos de WOS, 1312 de Scopus y 41 de las revistas españolas anteriormente citadas). Este resultado fue depurado eliminando artículos duplicados, obteniéndose un total de 1537 trabajos (269 de WOS, 1234 de Scopus y 34 de las revistas españolas).

\section{Selección de artículos}

Los 1537 artículos obtenidos anteriormente fueron sometidos a un proceso de selección que se realizó en dos fases.

La primera se realizó a partir de la lectura del título y del resumen de cada documento, seleccionando los artículos que hacían referencia tanto a procesos de enseñanza-aprendizaje como a implicaciones educativas relacionadas con el agua. Para comprender mejor esta primera criba, veamos los siguientes ejemplos: el trabajo de Ahi (2017) pone de manifiesto que la investigación está dirigida al proceso de enseñanza-aprendizaje del ciclo del agua, tanto en el título como en el resumen del trabajo, y por lo tanto supera esta primera fase. En la misma línea, podemos citar el trabajo de Ben Zvi y Orion (2005a), cuyo título no hace referencia al agua, pero sí su resumen, en el que se indica que el estudio realizado se centra en el ciclo del agua. Por el contrario, el estudio de Miller, Manz, Russ, Stroupe y Berland (2018), que aportó la búsqueda en WOS, indicó que el artículo no cumplía los criterios para formar parte de la selección, pues la investigación no afecta a los procesos de enseñanza-aprendizaje del agua sino que ésta se usa como excusa para la creación del constructo de «agencia epistémica».

Tras esta primera criba, se obtuvieron 38 artículos de WOS, 64 artículos de Scopus y 24 de las principales revistas españolas del área; tras la eliminación de duplicados, se obtuvo un total de 108 trabajos, el 7,02\% de los 1537 iniciales.

La segunda fase de la selección se realizó a través de la lectura de los textos completos. En este caso, el artículo debía contener desde un punto de vista tanto conceptual como metodológico elementos directamente relacionados con la enseñanza y/o aprendizaje del agua, de modo que pudiera contribuir a dar respuesta a las preguntas de investigación planteadas. A modo de ejemplo, el artículo de Agraso y Jiménez (2003) «Percepción de los problemas ambientales por el alumnado: los recursos naturales», que pasó la primera selección, tras la lectura completa fue descartado porque la investigación está centrada en la percepción del estudiantado sobre los problemas medioambientales en general, pero no concretamente en el agua.

Tras esta segunda fase, se obtuvieron 26 artículos de WOS, 31 de Scopus y 19 artículos de las revistas españolas. Eliminando repeticiones, la base de datos de la que se nutre esta revisión bibliográfica resulta finalmente formada por 47 artículos (el 43,52\% de los 108 trabajos potencialmente relevantes).

\section{Análisis}

De cada documento se extrajeron como datos bibliográficos: autores, nacionalidad -país al que pertenece la muestra de investigación y, en su ausencia, el autor principal-, año de publicación, título de la revista, y tipo de investigación (revisión bibliográfica, diagnóstico, intervención didáctica). 


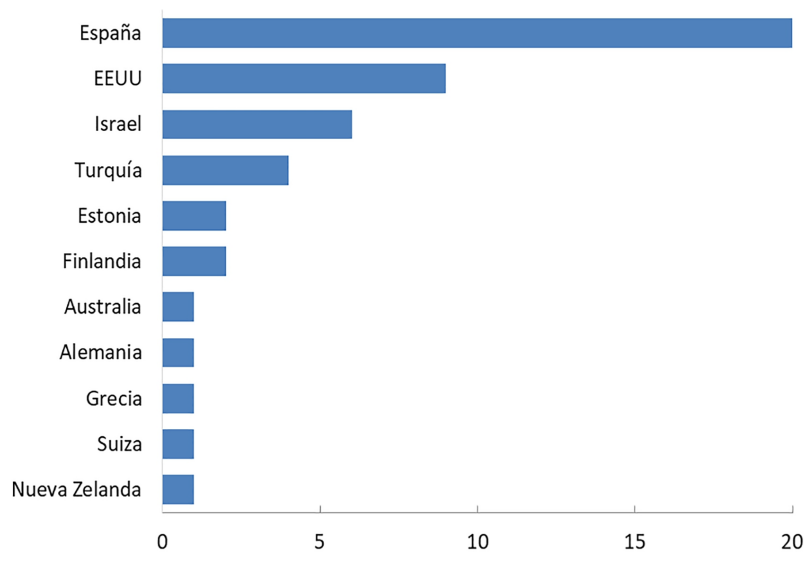

Figura 2. Distribución de artículos por nacionalidad
Se han contabilizado un total de 86 autores de 11 nacionalidades diferentes, distribuidos en 22 revistas. En la figura 2, se puede observar el número de artículos publicados en cada país.

De los 47 artículos, 34 fueron publicados en la segunda década, periodo 2010-2018, frente a 13 de la primera 2000-2009.

La distribución por etapas educativas es: 8\% para Infantil, 40\% para Primaria; 33\% para Secundaria; $11 \%$ para Bachillerato y $6 \%$ en Universidad.

En la figura 3 se muestra la distribución de los artículos en las distintas revistas. El mayor número de artículos se encuentra en la revista Journal of Research in Science Teaching con un total de seis artículos, seguido de tres revistas españolas con cinco artículos cada una: Alambique, Enseñanza de las Ciencias y Enseñanza de las Ciencias de la Tierra. Le siguen International Journal of Science Education, International Research in Geographical and Environmental Education y Journal of Geoscience Education con tres artículos; e Investigación en la Escuela, así como International Journal of Environmental and Science Education con dos. El resto de las revistas están representadas con un único artículo. Por lo que podemos ver, aunque el 72,3\% de los artículos están recogidos en tan solo nueve revistas, la dispersión es elevada, lo que está asociado al carácter interdisciplinar del agua.

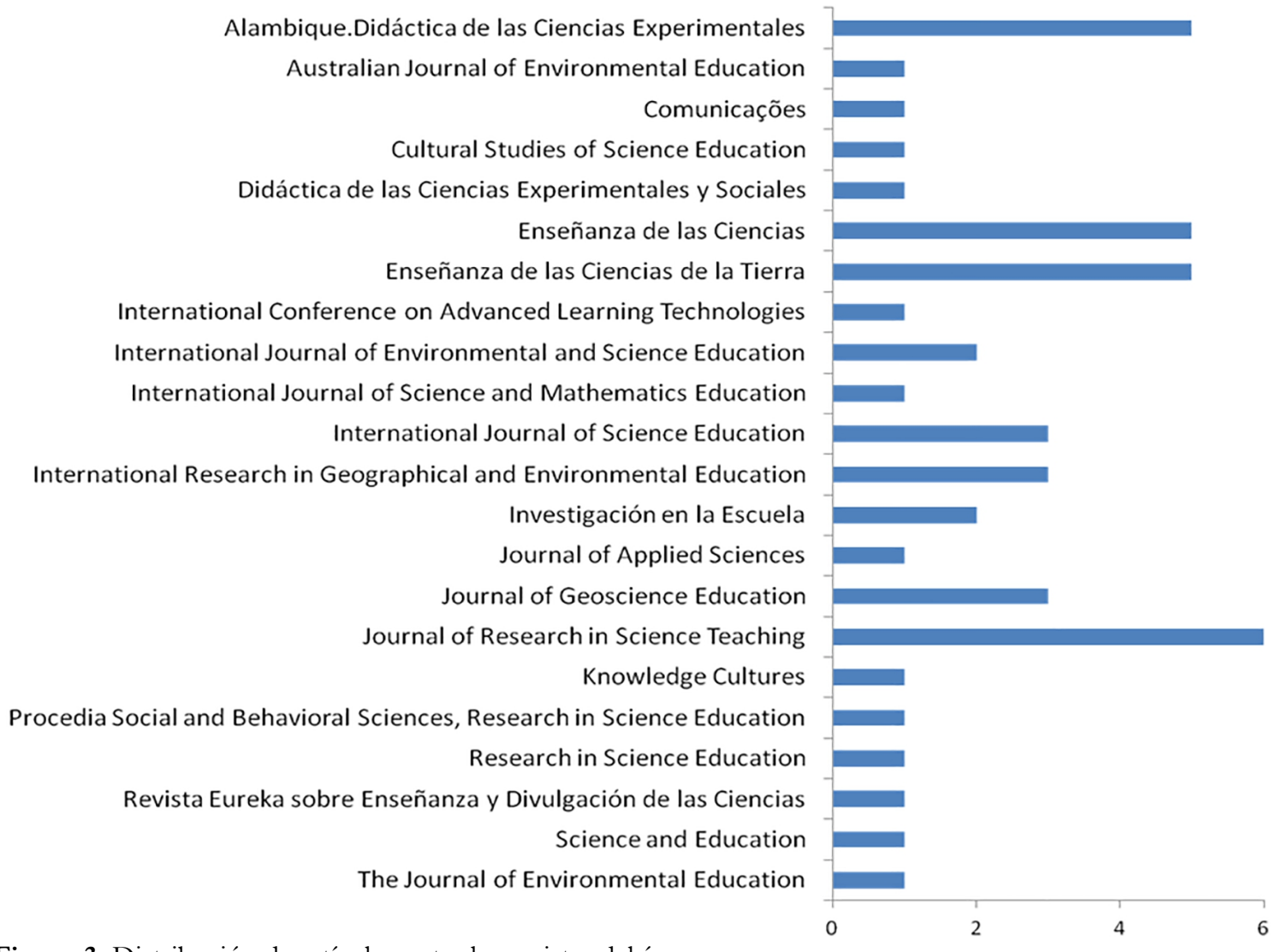

Figura 3. Distribución de artículos entre las revistas del área 
En cuanto al tipo de investigación se identifican una revisión bibliográfica, 16 estudios de diagnóstico y 30 intervenciones didácticas.

Para realizar el análisis de las temáticas tratadas en cada artículo seleccionado, se decidió partir de los contenidos curriculares relacionados con el agua en la legislación española vigente. Para ello, siguiendo a Marcén (2006), los contenidos curriculares se agruparon en tres bloques: los que se refieren a las características fisico-químicas del agua, los que aluden a la interacción del agua con el medio físico y aquéllos en los que la interacción implica al conjunto de seres vivos incluido el hombre. En la tabla 1 se muestra la distribución de los contenidos curriculares a través de las sucesivas etapas de enseñanza, según los decretos nacionales (MEC 2007; 2014; 2015).

Tabla 1. Contenidos del agua en el currículum español

\begin{tabular}{|c|c|c|c|c|c|c|}
\hline \multirow{2}{*}{$\begin{array}{l}\text { Título } \\
\text { Cabecera de tabla }\end{array}$} & \multirow{2}{*}{$\begin{array}{c}\text { INFANTIL } \\
3^{\circ}-6^{\circ}\end{array}$} & \multirow{2}{*}{$\frac{\text { PRIMARIA }}{1^{\circ}-6^{\circ}}$} & \multicolumn{2}{|c|}{ SECUNDARIA } & \multicolumn{2}{|c|}{ BACHILLERATO } \\
\hline & & & $1^{\circ}-3^{\circ}$ & $4^{\circ}$ & $1^{\mathrm{o}}$ & $2^{\circ}$ \\
\hline \multicolumn{7}{|l|}{ CARACTERÍSTICAS FISICO-QUÍMICAS } \\
\hline Cambios de estado y estados de agregación & $\mathrm{X}$ & $\mathrm{X}$ & $\mathrm{X}$ & & & \\
\hline Estructura y composición & & & & & $\mathrm{X}$ & $\mathrm{X}$ \\
\hline Precipitaciones & $\mathrm{X}$ & $\mathrm{X}$ & & & & $\mathrm{X}$ \\
\hline Propiedades & & & $\mathrm{X}$ & & $\mathrm{X}$ & $\mathrm{X}$ \\
\hline Tipos de agua & & & $\mathrm{X}$ & & & \\
\hline \multicolumn{7}{|c|}{ RELACIÓN DEL AGUA CON EL MEDIO FÍSICO } \\
\hline Acción geológica del mar/glaciales & & & $\mathrm{X}$ & & & $\mathrm{X}$ \\
\hline Aguas superficiales dulces y marinas & $\mathrm{X}$ & $\mathrm{X}$ & $\mathrm{X}$ & & & $\mathrm{X}$ \\
\hline Aguas subterráneas & & $\mathrm{X}$ & $\mathrm{X}$ & & & $\mathrm{X}$ \\
\hline Ciclo natural del agua & & $\mathrm{X}$ & $\mathrm{X}$ & & & $\mathrm{X}$ \\
\hline Configuración del relieve/paisaje & & $\mathrm{X}$ & $\mathrm{X}$ & & & $\mathrm{X}$ \\
\hline Cuencas hidrográficas y manantiales & & $\mathrm{X}$ & $\mathrm{X}$ & & & $\mathrm{X}$ \\
\hline Distribución del agua en la Tierra & & $\mathrm{X}$ & $\mathrm{X}$ & & & $\mathrm{X}$ \\
\hline Efectos medioambientales & & $\mathrm{X}$ & $\mathrm{X}$ & & & \\
\hline Hidrosfera & & $\mathrm{X}$ & $\mathrm{X}$ & $\mathrm{X}$ & & \\
\hline Riesgo geológico & & & $\mathrm{X}$ & & & $\mathrm{X}$ \\
\hline \multicolumn{7}{|c|}{ RELACIÓN DEL AGUA CON LOS SERES VIVOS } \\
\hline Agua y salud & $\mathrm{X}$ & $\mathrm{X}$ & $\mathrm{X}$ & & $\mathrm{X}$ & \\
\hline Agua y seres vivos & $\mathrm{X}$ & $\mathrm{X}$ & $\mathrm{X}$ & $\mathrm{X}$ & $\mathrm{X}$ & \\
\hline Ahorro & & $\mathrm{X}$ & $\mathrm{X}$ & $\mathrm{X}$ & & $\mathrm{X}$ \\
\hline Aprovechamiento de los recursos hídricos & & $\mathrm{X}$ & $\mathrm{X}$ & $\mathrm{X}$ & & $\mathrm{X}$ \\
\hline Calidad del agua & & & & & & $\mathrm{X}$ \\
\hline Consumo & & $\mathrm{X}$ & $\mathrm{X}$ & $\mathrm{X}$ & & \\
\hline Contaminación & & $\mathrm{X}$ & $\mathrm{X}$ & $\mathrm{X}$ & & $\mathrm{X}$ \\
\hline Gestión & & $\mathrm{X}$ & $\mathrm{X}$ & $\mathrm{X}$ & & \\
\hline Importancia para la existencia de vida & $\mathrm{X}$ & $\mathrm{X}$ & $\mathrm{X}$ & & $\mathrm{X}$ & \\
\hline Sistemas de potabilización/depuración & & & & $\mathrm{X}$ & & $\mathrm{X}$ \\
\hline Usos cotidianos & & & $\mathrm{X}$ & & & $\mathrm{X}$ \\
\hline Uso urbano del agua & & & $\mathrm{X}$ & $\mathrm{X}$ & & \\
\hline
\end{tabular}


A cada artículo se le asignó una cifra que expresaba la relación de su contenido con cada uno de los tópicos curriculares relacionados con el agua, utilizando la siguiente escala numérica:

1. Contenido principal: el contenido aparece en el título y/o en las palabras clave del artículo y/o en el resumen.

2. Contenido secundario investigado: el contenido no está incluido en el título ni en las palabras clave ni en el resumen, pero es objeto de investigación en el artículo.

3. Contenido secundario no investigado: el contenido no es objeto de investigación, pero es citado y referenciado bibliográficamente.

4. Contenido secundario no investigado ni referenciado: el contenido no es objeto de investigación, y es citado aunque no se referencia bibliográficamente.

Por ejemplo, el artículo de Cano (2007) adquirió un 1 en contaminación, por ser el contenido principal investigado; un 2 en el ciclo urbano del agua, consumo, sistemas de potabilización/ depuración y usos cotidianos, por ser contenidos también investigados en el artículo, aunque no como contenidos principales; por último, obtiene un 4 en aguas superficiales dulces y marinas y en agua $y$ seres vivos, al ser contenidos citados en el transcurso del artículo, aunque sin referencias bibliográficas.

La asignación fue realizada por las tres investigadoras firmantes, primero de modo independiente $\mathrm{y}$, posteriormente, se contrastaron los resultados. Este proceso se reiteró modificando la definición de la escala numérica hasta que su arbitrariedad fuera minimizada y el grado de acuerdo de las investigadoras alcanzara el 95\%.

\section{Resultados}

En la tabla 2 se presenta una síntesis de los resultados obtenidos en cuanto a contenidos se refiere. Un dato llamativo es que, de los tres bloques de contenidos, el más investigado como contenido principal es el de Relación del Agua con el Medio fisico, con 30 artículos, frente a 13 para la Relación del Agua con los Seres Vivos. Esta distribución puede estar reflejando una tendencia en las investigaciones didácticas a ocuparse preferentemente de los contenidos físico-químicos (ciclo natural del agua, cuencas...) frente a los contenidos medioambientales (gestión, ciclo urbano del agua, depuración...), cuyas investigaciones son necesarias para ayudar al alumnado a comprender el papel de los humanos en la disponibilidad de los recursos hídricos y avanzar en sus concepciones desde una concepción local y subjetiva del agua a otra más extensa en el espacio y en el tiempo y más compartida, descentrada y objetiva.

Por otro lado, de los 30 artículos dedicados a la Relación del Agua con el Medio Físico, 20 estudian el ciclo del agua como contenido principal, seguido a gran distancia de otros contenidos. Se confirma, también desde la perspectiva de la investigación didáctica, la importancia del ciclo del agua como contenido estrella de esta temática.

En cuanto a los contenidos secundarios, los más estudiados son los relacionados con cambios de estado y estados de agregación, con 29 artículos, seguidos de aguas superficiales y subterráneas, con 23 y 24 artículos respectivamente, todos ellos contenidos íntimamente relacionados con el ciclo del agua. 
Tabla 2. Distribución de artículos según la relación de su contenido con cada uno de los tópicos curriculares relacionados con el agua

\begin{tabular}{|c|c|c|c|c|c|}
\hline & & \multicolumn{4}{|c|}{$\begin{array}{l}\mathrm{N}^{\circ} \text { de artículos por } \\
\text { categoría de clasificación }\end{array}$} \\
\hline \multicolumn{2}{|r|}{ CONTENIDOS } & 1 & 2 & 3 & 4 \\
\hline \multirow{5}{*}{$\begin{array}{l}\text { CARACTERÍSTICAS } \\
\text { FISICO-QUÍMICAS }\end{array}$} & Cambios de estado y estados de agregación & 1 & 29 & 2 & 2 \\
\hline & Estructura y composición & - & 4 & 2 & 4 \\
\hline & Precipitaciones & 2 & 15 & 1 & 3 \\
\hline & Propiedades & - & 12 & 2 & 7 \\
\hline & Tipos de agua & - & 5 & 2 & 3 \\
\hline & TOTAL & 3 & 65 & 9 & 19 \\
\hline \multirow{10}{*}{$\begin{array}{l}\text { RELACIÓN DEL AGUA } \\
\text { CON EL MEDIO } \\
\text { FÍSICO }\end{array}$} & Acción geológica del mar/glaciales & - & 5 & 1 & 1 \\
\hline & Aguas superficiales dulces y marinas & - & 23 & 2 & 1 \\
\hline & Aguas subterráneas & 4 & 24 & 2 & 0 \\
\hline & Ciclo natural del agua & 20 & 15 & 4 & 1 \\
\hline & Configuración del relieve/paisaje & - & 2 & - & 1 \\
\hline & Cuencas hidrográficas y manantiales & 5 & 14 & 2 & 1 \\
\hline & Distribución del agua en la Tierra & - & 8 & 1 & 2 \\
\hline & Efectos medioambientales & - & 1 & - & 1 \\
\hline & Hidrosfera & - & 7 & 3 & - \\
\hline & Riesgos geológicos & 1 & 3 & 1 & 1 \\
\hline & TOTAL & 30 & 102 & 16 & 9 \\
\hline \multirow{12}{*}{$\begin{array}{l}\text { RELACIÓN DEL AGUA } \\
\text { CON LOS SERES } \\
\text { VIVOS }\end{array}$} & Agua y salud & - & 3 & 1 & - \\
\hline & Agua y seres vivos & - & 10 & 3 & 3 \\
\hline & Ahorro & - & 7 & 3 & - \\
\hline & Aprovechamiento recursos hídricos & - & 9 & 3 & 3 \\
\hline & Calidad del agua & - & 7 & 3 & 2 \\
\hline & Consumo & 2 & 12 & 1 & - \\
\hline & Contaminación & 5 & 15 & 3 & 1 \\
\hline & Gestión & 4 & 6 & 3 & 1 \\
\hline & Importancia para la existencia de vida & - & 6 & 3 & 11 \\
\hline & Sistemas de potabilización/depuración & 1 & 14 & 1 & 1 \\
\hline & Usos cotidianos & - & 6 & 1 & 1 \\
\hline & Uso urbano del agua & 1 & 15 & 1 & - \\
\hline & TOTAL & 13 & 110 & 26 & 22 \\
\hline
\end{tabular}

A continuación se sintetizan las características de los artículos agrupados por contenidos curriculares, seleccionando únicamente los que han sido trabajados como contenido principal.

\section{Cambios de estado y estados de agregación}

Solo se ha encontrado un trabajo que trata este contenido como principal en el periodo de tiempo analizado, frente a los 29 que lo trabajan como secundario.

El trabajo de Cruz-Guzmán, García-Carmona y Criado (2017) es una intervención didáctica basada en la indagación para abordar los cambios de estado del agua y de otras sustancias (chocolate y un polo flash). La intervención se llevó a cabo durante dos cursos académicos 
consecutivos con escolares de primer y segundo ciclo de infantil. Los autores destacan los beneficios del método indagativo para esta etapa educativa y las dificultades que las niñas y los niños tienen para comprender la presencia de agua en el aire, así como las asociadas a la extrapolación de los cambios de estado del agua a otras sustancias.

\section{Precipitaciones}

En este apartado se recogen los artículos que tratan sobre la lluvia y la formación de las nubes (y el arco iris). Tan solo dos investigaciones tratan este contenido como principal, siendo más numerosos los artículos que lo estudian como secundario, un total de 15. Ambos trabajos son diagnósticos. Sus resultados pusieron de manifiesto la dificultad del alumnado para explicar los procesos implicados en la formación de las nubes y la lluvia recurriendo a concepciones alternativas. Una de las respuestas más recurrentes es que las nubes están hechas de vapor de agua. Según Malleus, Kikas y Marken (2017) esta respuesta es más común en estudiantes de primaria que de infantil, ya que en esa etapa se enseña la condensación y la evaporación y, cómo este último concepto es más comprensible, el alumnado tiende a incorporarlo para argumentar que las nubes están formadas por vapor de agua y la lluvia por condensación de estas. Los autores consideran de gran importancia que los docentes promuevan una mayor integración del conocimiento científico con la experiencia personal en estas etapas para obtener una mejor comprensión de los conocimientos. En la investigación de Malleus, Kikas y Kruus (2016) se observó que el profesorado subestima el conocimiento del alumnado de 8 a 9 años y sobrestima el del alumnado de 9 a 12 años, lo que se explica porque en estas últimas edades ya han realizado sus intervenciones y sobrevaloran los efectos de sus enseñanzas.

\section{Aguas subterráneas}

Las aguas subterráneas son ampliamente contempladas como contenido secundario, en un total de 24 artículos. Sin embargo, en la literatura analizada tan sólo cuatro trabajos tienen como objetivo principal de su investigación este contenido.

Los estudios de diagnóstico de Dickerson y Dawkins (2004) y de Fernández y González (2010) tienen como objetivo identificar los conocimientos alternativos y esquemas de conocimiento sobre el agua subterránea respectivamente. Dickerson y Dawkins (2004) analizaron las concepciones alternativas sobre las aguas subterráneas en estudiantes de secundaria estadounidenses matriculados en un curso optativo de Ciencias de la Tierra, en términos del conflicto lingüístico entre su lengua materna y el lenguaje científico. Para los autores, otra fuente de conflictos surge de la invisibilidad de estas aguas, así como de las limitaciones del razonamiento espacial del alumnado. Fernández y González (2010) analizaron los esquemas de conocimiento en estudiantes universitarios españoles, diferenciando el bachillerato cursado, sobre el papel del agua subterránea para explicar la procedencia del agua de los ríos tras meses sin precipitaciones. Tras el análisis de los resultados, los investigadores destacan los problemas del alumnado en cuanto a la terminología, funcionamiento y localización de las aguas subterráneas, demostrando tener una importante incultura sobre hidrogeología, sólo superada en los que habían cursado dicha asignatura, destacando la importancia de la toma de conciencia del profesorado sobre esta temática. En un trabajo posterior, Fernández y González (2013), plantean una propuesta didáctica basada en la investigación dirigida, para intentar que los estudiantes de secundaria superen la dificultad de entendimiento relativa a la descarga subterránea que alimenta el caudal de los ríos.

En la intervención didáctica de Calvo et al. (2007) se describe un proceso de trabajo con modelos para el estudio de las aguas subterráneas. La intervención consiste en la construcción de modelos simulando acuíferos en diferentes formas geológicas. Sus autores concluyen que 
ello ayuda al alumnado a construir modelos mentales más avanzados sobre las aguas subterráneas.

\section{Ciclo natural del agua}

El ciclo del agua es el contenido más estudiado en el período de tiempo analizado. De hecho, se han encontrado 20 artículos que lo tratan como contenido principal, y un total de 15 que lo incluyen como contenido secundario. De las 20 investigaciones que tienen este contenido como objetivo principal de su investigación encontramos una revisión bibliográfica, seis estudios de diagnóstico y 13 intervenciones didácticas.

La revisión bibliográfica de Hernández (2014) pone de manifiesto la visión simple y errónea que se tiene del ciclo del agua, que además es reforzada por la mayoría de los libros de texto e ilustraciones. Según la autora, para una correcta interpretación del ciclo natural del agua, es importante conocer el balance entre evaporación y precipitación en zonas continentales y oceánicas, el papel que tienen los seres vivos en la evapotranspiración y considerar a las aguas subterráneas como nuestra principal fuente de abastecimiento.

Los estudios de diagnóstico que se han llevado a cabo en el período de tiempo estudiado, ponen de manifiesto que el alumnado de las diferentes etapas educativas analizadas y de diferentes países, poseen un conocimiento incompleto sobre el ciclo natural del agua. Son capaces de comprender algunos procesos del ciclo pero la mayoría carece de las percepciones dinámicas, cíclicas y sistémicas del mismo (Ben-Zvi y Orion 2005a; Cardak 2009). En concreto, son pocos los que hacen referencia a las aguas subterráneas, aguas residuales y seres vivos (Cardak 2009; Shepardson, Wee, Priddy, Schellenberger, y Harbor 2009), es decir, se centran fundamentalmente en los procesos de transformación del agua, pero no identifican, o lo hacen de forma incompleta, la circulación subterránea del agua y el impacto que las actividades humanas pueden tener en el ciclo natural del agua, lo que indica que el conocimiento que poseen del ciclo del agua está limitado a un flujo de agua a través de sistemas sin vida (Cardak 2009).

El análisis de las imágenes del ciclo del agua que aparecen en las editoriales españolas de mayor prestigio, analizadas por Reyero et al. (2007), pone de manifiesto la existencia de errores conceptuales y la omisión o simplificación de algunas partes del ciclo, que se corresponden con la imagen incompleta del ciclo que presenta el alumnado (Cardak 2009; Reyero et al. 2007). Estos resultados acentúan la importancia de conocer las dificultades e ideas alternativas que el alumnado tiene sobre el ciclo natural del agua para reforzar las ilustraciones de los manuales escolares.

La mayoría del estudiantado no identifica el impacto de las actividades humanas sobre el ciclo del agua (Ben-Zvi, Eshach, Orion y Alamour 2012; Cardak 2009; Shepardson et al. 2009), por lo que no son capaces de conectarlo con su entorno. Además, el estudio sobre la construcción de modelos espontáneos y no espontáneos conectados con las diferentes culturas y estilos de vida llevado a cabo por Ben-Zvi et al. (2012), pone de manifiesto que los modelos mentales sobre el ciclo del agua son más complejos en estudiantes beduinos que en estudiantes judíos de una pequeña ciudad provincial, lo cual demuestra la importancia de relacionar el ciclo del agua con el entorno del estudiante para evitar un conocimiento parcial.

La investigación de Romine, Schaffer y Barrow (2015) describe el desarrollo y la validación de una prueba de diagnóstico de concepciones alternativas en estudiantes universitarios sobre el ciclo del agua. El análisis del cuestionario evidenció la ineficacia de los cursos de ciencias recibidos para conseguir que el alumnado integre significativamente las conceptualizaciones científicas sobre el ciclo del agua. 
Las intervenciones didácticas presentes en la literatura abordada están publicadas mayoritariamente en la segunda década analizada y tienen como objetivo mejorar la comprensión del ciclo natural del agua y solventar las dificultades de enseñanza-aprendizaje que este complejo sistema presenta para el alumnado y que son diagnosticadas fundamentalmente en la primera década del estudio.

Las investigaciones de Ben-Zvi y Orion (2005b, 2010a) están basadas en el estudio de las bondades de una unidad didáctica denominada Planeta Azul, que incluye 45 horas de investigación en el laboratorio y de aprendizaje al aire libre, con actividades que se desarrollaron para ayudar al alumnado de secundaria a adquirir sistemas de pensamiento en el contexto del ciclo del agua. Los autores ponen de manifiesto las dificultades de la adquisición del pensamiento sistémico y proponen para su desarrollo una estructura piramidal jerárquica, cuyo progreso se debe a dos factores, las habilidades cognitivas individuales de cada estudiante, y su nivel de participación en las actividades de integración del conocimiento durante su aprendizaje. En la segunda investigación, analizan los resultados de aprendizaje de cuatro estudiantes que habían realizado la unidad didáctica Planeta Azul y, confirman con datos recogidos incluso seis años después, que el desarrollo del pensamiento sistémico se produce de forma jerárquica. El trabajo de Ben-Zvi y Orion (2010b) es una intervención didáctica basada en la indagación para desarrollar las habilidades del pensamiento sistémico en la etapa de primaria. La instrucción incluyó simulaciones y experimentos de laboratorio, interacción directa con componentes y procesos del ciclo del agua en el entorno de aprendizaje al aire libre y actividades de integración del conocimiento. Los autores destacan que la instrucción por indagación realizada contribuyó a que el alumnado de primaria a su corta edad fuera capaz de desarrollar las habilidades de pensamiento sistémico.

Las investigaciones de Nebot (2007); Nebot y Márquez (2014); y Otrel-Cass (2017) se centran en la simulación de los procesos implicados en el ciclo natural del agua a través de la modelización.

En el trabajo de Nebot (2007) se explica con detalle el proceso de construcción de una maqueta, utilizando para ello una garrafa de agua, para estudiar los diferentes procesos del ciclo natural del agua así como diversas situaciones relacionadas con la contaminación y la salinización de un acuífero. Nebot y Márquez (2014) consideran que trabajar con la modelización ayuda al alumnado a interpretar muchos de los procesos del ciclo natural del agua y relacionar dichos conceptos con el modelo real que representan, destacando que este tipo de instrucción permite entender los procesos dinámicos del ciclo, en especial los procesos no visibles, ya sean por la observación directa (aguas subterráneas) o porque son inferidos (evaporación y condensación). En el trabajo de Otrel-Cass (2017) la modelización forma parte de una unidad didáctica de tres semanas de duración en la que el autor utiliza diversos materiales visuales a través de los cuales evidenciaron el grado de comprensión que adquirieron sobre el ciclo natural del agua.

Las intervenciones de Márquez y Bach (2007); Roca, Márquez y Sanmartí (2013); Castelltort (2015) y Ahi (2017) tienen como propósito conocer la evolución que se produce en los modelos mentales del alumnado sobre el ciclo natural del agua después de llevar a cabo el proceso de enseñanza-aprendizaje.

Márquez y Bach (2007) trabajan con estudiantes de 12 a 14 años a los que solicitan diagramas del ciclo del agua en tres momentos didácticos (al inicio, durante la intervención y un año después). Concluyen que, tras la instrucción, el alumnado plasmó en sus diagramas un mayor número de componentes estáticos (almacenes de agua, como glaciares y cimas de montaña) y dinámicos (cambios de lugar y de estado del agua). La intervención didáctica de Roca et al. (2013) consiste en una unidad didáctica de 10 horas de duración que promueve el 
planteamiento de preguntas por parte del alumnado, así como la reflexión sobre la importancia de estas, además de perseguir los objetivos de aprendizaje característicos del ciclo natural del agua. El análisis de las preguntas formuladas por el estudiantado permite identificar a los autores los modelos del ciclo natural que presentan; estos modelos van desde una visión estática y atomizada del sistema (preguntan por los fenómenos del ciclo, pero de forma aislada y no tienen en cuenta ni la fase atmosférica ni la subterránea) hasta una visión más dinámica y compleja (preguntan por las distintas fases del ciclo). El trabajo de Castelltort (2015) es un estudio de casos realizado con tres escuelas de educación primaria, participantes del Programa Agenda 21 Escolar de Barcelona, para ayudar al alumnado a actuar en la construcción de un futuro más sostenible. Los resultados respecto al ciclo natural del agua evidenciaron que el estudiantado no solo asimilaba mejor los procesos y cambios de estado implicados en el ciclo natural del agua, sino que sus modelos llegaban a ser más complejos y completos, con la inclusión de la circulación subterránea. La intervención didáctica de Ahi (2017) gira en torno a un proceso experimental en el que se utiliza la técnica talking drawings que consistió en ilustrar las ideas que tienen los escolares de la etapa de infantil sobre el ciclo natural del agua a través de dibujos que posteriormente tienen que explicar al conjunto de compañeros. El autor destaca las ventajas de esta técnica para el desarrollo de los modelos mentales, incluso en esta etapa educativa.

En la misma línea que en los anteriores trabajos, los de Forbes, Zangori y Schwarz (2015) y Zangori, Forbes y Schwarz (2015) indagan en el efecto de unidades didácticas sobre el ciclo natural del agua, en este caso para la etapa de primaria y caracterizadas por el uso de tareas o actividades en las que se emplea el modelado por andamiaje. En ambos trabajos, se fomenta la construcción de modelos del ciclo natural del agua por parte de los escolares a través de actividades andamiadas en las que pueden utilizar diferentes elementos (bocetos, flechas, palabras, números) para construir y revisar sus modelos. Tras la instrucción con andamiaje, el alumnado realizó representaciones más sofisticadas, en las que enfatizaban los componentes invisibles del ciclo del agua. Los autores concluyen que las tareas con modelado ofrecen una oportunidad al estudiantado para visualizar sus ideas sobre el ciclo dentro de los objetivos de aprendizaje marcados.

Todas las investigaciones anteriormente citadas tratan el ciclo natural del agua de manera integral; sin embargo, el trabajo de Bratitsis, Bardanika y Ioannou (2017) se centra en los cambios de estados del agua que se producen en el ciclo (evaporación, condensación, fusión, solidificación). En este trabajo, se realiza una intervención didáctica con estudiantes de primaria basada en la realidad aumentada en la que se desarrolla una historia digital utilizando el software ENTITI, cuyo personaje principal es una gota de agua que experimenta una aventura y necesita la ayuda de los escolares para recrear su historia. Los autores subrayan que el uso de la realidad aumentada permitió a los escolares comprender mejor los cambios de estado (aunque presentaron dificultades con respecto al proceso de condensación), recrearlos y secuenciarlos correctamente de forma cíclica y por tanto, alcanzar la comprensión de que el agua no desaparece pues es reciclada a través de los diferentes procesos del ciclo.

\section{Cuencas hidrográficas y manantiales}

Tan sólo cinco investigaciones tienen este contenido como principal, de las cuales, cuatro versan sobre las cuencas hidrográficas y una sobre los manantiales.

El objetivo del estudio de diagnóstico de Shepardson et al. (2005) es conocer las ideas alternativas del alumnado de secundaria sobre las cuencas hidrográficas y cómo estas ideas cambian a través de las etapas educativas. Comprobaron que sólo representan las cuencas en áreas naturales y conectadas con los ríos, tal y como aparecen típicamente dibujadas en los 
libros de textos y en ningún caso las relacionan con otras fuentes de agua ni con fuentes de contaminación.

Las intervenciones didácticas de Nebot (2007) y Nebot y Márquez (2014) consisten en una secuencia didáctica cuya actividad central se basa en la elaboración de una maqueta para estudiar las cuencas hidrográficas y los procesos implicados en el ciclo del agua. En ambos trabajos se comprobó que la realización de maquetas favorecía la comprensión e interpretación de los fenómenos implicados en el ciclo del agua y la relación entre ellos.

La intervención didáctica de Endreny (2010) se basa en una unidad didáctica sobre cuencas hidrográficas contextualizada en el lugar de residencia del alumnado de 11 años, con el objetivo de que lleguen a comprender cómo afecta la actividad humana a la calidad del agua de la cuenca hidrográfica. Aunque el objetivo fue alcanzado, se encontraron dificultades con conceptos que no se pudieron ejemplificar como la infiltración o la escorrentía. Por ello, según el autor, el estudio proporcionó una evidencia clara de que el fomento del aprendizaje basado en el entorno permite un mejor desarrollo del conocimiento del alumnado.

Reinfried, Aeschbacher, Kienzler y Tempelmann (2015) destacan que los manantiales de agua son un concepto hidrológico importante porque forman una interfaz entre los subsistemas subterráneos y superficiales del ciclo hidrológico. Diseñaron una intervención didáctica para su enseñanza a estudiantes de primaria, basada en la Reconstrucción Educativa (MER), un enfoque constructivista que consiste en el diseño de secuencias de aprendizaje con el fin de cambiar las ideas intuitivas del alumnado. En base a las concepciones iniciales obtenidas y los conceptos científicos sobre los manantiales, los autores diseñaron un conjunto de categorías adecuadas para el grupo de edad estudiado. Los resultados indicaron un aumento de los conocimientos del alumnado sobre manantiales incluso dos meses después de la finalización de la intervención.

\section{Riesgos geológicos}

Dentro de esta categoría se incluyen los riesgos geológicos provocados tanto por el exceso como por la escasez de agua. Son pocos los trabajos dedicados a este contenido, concretamente se ha encontrado uno que lo estudia como contenido principal y tres como secundario.

La investigación de Sammel, McMartin y Arbuthnott (2018) analiza el contenido curricular genérico relacionado con el agua y con los riesgos geológicos que provoca, en el currículum de Australia y Canadá, regiones geográficamente vulnerables. El análisis de los planes de estudio de la educación formal de ambos países evidenció que, aunque proporcionan un marco sólido para comprender la naturaleza hidrológica del agua, no permiten llegar a entender la relación del agua dulce con los sistemas sociales, ni la influencia cultural, la gestión ambiental y los procesos de seguridad y riesgos geológicos relacionados con la misma. Para abordar esta situación los autores proponen que los planes de estudios incorporen investigaciones sobre las sequías e inundaciones y prácticas locales e inclusivas en el entorno del alumnado, con el objetivo de que entiendan la ciencia del agua y las complejas interacciones sociopolíticas que gobiernan su gestión.

\section{Consumo}

Sólo dos investigaciones trabajan este contenido como principal en el periodo de tiempo analizado, aunque son más numerosas las que lo hacen como contenido secundario, un total de 12.

La intervención didáctica de Fernández y Solís (2011) se detalla en el apartado de contaminación y al igual que para este contenido los autores consideran que en el proceso de 
construcción del conocimiento, el alumnado es capaz de realizar con mucha facilidad, evoluciones y retornos entre los niveles de categorías de análisis del conocimiento.

La intervención didáctica de Çoban, Akpinar, Küçükcankurtaran, Yildiz y Ergin (2011) tiene como objetivo dar a conocer los resultados obtenidos después de llevar a cabo un Taller sobre el Agua con estudiantes de 11 a 14 años de Turquía. Los resultados mostraron que los materiales didácticos empleados fueron adecuados para esta etapa educativa promoviendo cambios en las áreas de aprendizaje cognitivo y afectivo. De hecho, el alumnado llevó a cabo un aprendizaje significativo y permanente sobre conceptos relacionados con el agua, mejoraron positivamente sus actitudes hacia el uso del agua y mostraron una mayor concienciación ambiental.

\section{Contaminación}

Se han encontrado cinco trabajos que incluyen este contenido como principal, una revisión bibliográfica, un estudio de diagnóstico y tres intervenciones didácticas, siendo un total de 15 las que lo incluyen como secundario.

En la revisión bibliográfica de Hernández (2014) se destaca que aunque este contenido es ampliamente tratado en la enseñanza obligatoria, no existen garantías de que se esté llevando a cabo una correcta educación medioambiental sobre la problemática del agua. Considera necesario que el alumnado conozca los altos inconvenientes de los procesos de depuración (costes elevados, residuos que necesitan purificarse, que el agua tratada devuelta al medio no se puede reutilizar para consumo humano...) y que los vertederos son fuentes de contaminación potenciales tanto de las aguas superficiales como de las aguas subterráneas.

La investigación de Kiryak y Çalik (2018) está basada en un estudio diagnóstico del modelo de construcción del conocimiento compartido (Common Knowledge Construction Model, CKCM en adelante) y su influencia en la comprensión conceptual del alumnado sobre contaminación del agua. Los autores destacan que el CKCM ayudó a mejorar la comprensión del alumnado sobre los contenidos, ya que antes de la intervención presentaban un conocimiento fragmentado de la contaminación ambiental del aire, suelo y agua, y eran incapaces de vincular estos conocimientos entre sí.

El objetivo de las intervenciones de Cano (2007) y de Fernández y Solís (2011) fue analizar el proceso de construcción significativo en relación con la contaminación (y el uso y consumo del agua). En la primera se desarrollan las hipótesis de progresión y en la segunda las categorías de análisis para la construcción del conocimiento significativo. Los resultados de estos trabajos evidencian que en el proceso de construcción del conocimiento, el alumnado es capaz de evolucionar desde un pensamiento simple a uno complejo, y que el retorno se produce con facilidad; sin embargo, los conocimientos adquiridos en un contexto, no se mantienen al cambiar de contexto y tampoco son fáciles de ser aplicados en otra materia; también se observó la dificultad del alumnado para entender el proceso de investigación y explicárselo de una forma clara a sus compañeros (Cano 2007).

La intervención realizada por Jaén, Esteve y de Pro (2014) propone el aprendizaje basado en problemas, centrado en la contaminación de las aguas del Mar Menor y el consecuente aumento de la población de medusas, para acercar al alumnado de secundaria a la comprensión de las repercusiones sociales de la ciencia y la tecnología en el problema de la contaminación de las aguas. Se pretende hacerlos reflexionar sobre las responsabilidades y que participen en las propuestas de soluciones. Los autores concluyen que en el marco de la LOMCE este tipo de propuestas no se favorecen, pero que son imprescindibles para un mejor aprendizaje del alumnado. 


\section{Gestión}

En este contenido se incluye gestión del agua y gestión sostenible. Se han encontrado cuatro trabajos que lo incluyen como contenido principal, todos ellos realizados en España, y seis que lo incluyen como contenido secundario.

En la revisión bibliográfica de Hernández (2014) respecto a la gestión sostenible, la autora analiza la formación que recibe el alumnado a lo largo de la enseñanza obligatoria con la intención de realizar una propuesta de síntesis de los contenidos que debería conocer todo ciudadano sobre el agua. Agrupa estos contenidos en «el agua en la naturaleza», y en «el agua como recurso».

La investigación de Jaén y Palop (2011) es un estudio diagnóstico de los conocimientos del alumnado de secundaria sobre la gestión del agua, la energía y los residuos. En relación con la gestión del agua, los autores sintetizan los conocimientos erróneos más habituales del alumnado sobre almacenes de agua dulce, sobre el acceso de la población al agua potable, sobre las instalaciones destinadas al agua en las ciudades, y destaca que la solución más frecuente propuesta por ellos al problema del agua es la construcción de más desalinizadoras o la realización de trasvases. Preguntaron también al profesorado sobre el origen de estas cuestiones, y la mayoría consideraba que las actividades realizadas para fomentar y motivar al alumnado en la conservación del medio ambiente son insuficientes, y que además debido a la falta de tiempo y de recursos los temas de medioambiente son los que generalmente se quedan sin impartir.

La investigación de Castelltort et al. (2014) es analizada en el apartado del uso urbano del agua. En lo referente a la gestión sostenible del agua, los resultados mostraron que las acciones que conoce y que practica el alumnado son muy repetidas socialmente, como cerrar el grifo, limitar el tiempo de la ducha, evitar los baños, ahorrar el agua y no hacen referencias a prácticas domésticas o relacionadas con la reutilización del agua. En cambio, tras la intervención llevada a cabo las ideas que presentaron fueron más amplias e incluían el ahorro del agua en el uso doméstico, así como en la escuela (que siguen siendo respuestas habituales), e incluyeron otras nuevas como utilizar poco papel higiénico, controlar el agua de riego del huerto, o reparar averías en cisternas, grifos y fuentes.

En el trabajo de Pascual (2014) se contempla una propuesta didáctica para introducir en el aula el tema de la gestión integral del agua. Dicha propuesta puede adaptarse a las diferentes etapas educativas, aunque los autores la recomiendan para bachillerato o los últimos cursos de la ESO. La finalidad de la propuesta es que a través de los diferentes escenarios que se puedan proponer, el alumnado sea capaz de deducir los efectos que se derivan de cada una de las acciones planteadas y consigan dar con la respuesta más adecuada. Esta propuesta permitiría al alumnado reflexionar sobre la necesidad de llevar a cabo una gestión sostenible frente al crecimiento actual de la demanda.

\section{Sistemas de potabilización/depuración}

Se incluyen en este contenido, tanto los sistemas de potabilización y desalinización del agua como la depuración o purificación de aguas residuales. Sólo se ha encontrado una investigación que tenga este contenido como principal, siendo más numerosas las investigaciones que lo incluyen como secundario, con un total de 14 artículos.

El trabajo de Köseoğlu, Soran y Storer (2009) es una intervención educativa sobre la depuración de las aguas residuales a través de un método de enseñanza conocido como estaciones de aprendizaje (Learning stations). Los autores analizan el conocimiento de cuatro estudiantes para indagar en la eficacia del método de enseñanza, y encuentran un fuerte 
incremento en la motivación, además de la mejora en la comprensión de los contenidos. Por otro lado destacan la necesidad de formar al profesorado a través de seminarios así como incluir estos métodos en la formación universitaria de los futuros docentes.

\section{Uso urbano del agua}

Si bien tan sólo una investigación tiene este contenido como principal, 15 trabajos lo incluyen como contenido secundario.

La intervención didáctica de Castelltort, Sanmartí y Pujol (2014) forma parte de un proyecto sobre el agua y el futuro, llevado a cabo con estudiantes de $4^{\circ}$ de primaria. A lo largo del proyecto se realizaron diferentes actividades que complementaban el trabajo en el aula y su entorno urbano. $\mathrm{Al}$ inicio del proyecto los autores observaron que los escolares desconocían la circulación del agua en su ciudad, posiblemente por la lejanía de las instalaciones implicadas de los centros urbanos. Tras la intervención didáctica, observaron una mejora importante en el conocimiento sobre el ciclo urbano por parte del alumnado, destacando la necesidad de aprovechar los recursos proporcionados por el entorno para enriquecer el proceso de enseñanza-aprendizaje, sobre todo con contenidos que se trabajan de manera insuficiente en la educación formal.

\section{Artículos sin contenido principal}

En este apartado hemos recogido todos los artículos que no presentan ningún contenido principal según la clasificación establecida al inicio de la revisión, bien porque sean muchos los contenidos trabajados y no destaque ninguno como principal, bien porque el objetivo del trabajo sea transversal (modelización, mapas conceptuales...) y los contenidos del agua sean medios y no fines en sí mismos.

En total se han incluido siete artículos entre los que se encuentran dos estudios de diagnósticos y cinco intervenciones didácticas.

El estudio de diagnóstico de Covitt, Gunckel y Anderson (2009) tiene como objetivo saber qué conocimientos tiene el alumnado sobre los sistemas ambientales a través de los cuales fluye el agua y cómo esta se conserva a través de los sistemas naturales y urbanos conectados. Tras el análisis de los resultados, los autores consideran necesario fomentar un currículum que favorezca una mayor comprensión y conexión entre los sistemas ambientales naturales y urbanos.

El estudio de diagnóstico de Gunckel et al. (2012) se centró en analizar la progresión de aprendizaje del alumnado sobre el agua y las sustancias del agua que se mueven a través de los sistemas atmosféricos, superficiales, subsuelos y los sistemas diseñados por el hombre. Los autores destacaron que pocos estudiantes fueron capaces de reconocer que los sistemas ambientales operan de acuerdo con las leyes físicas de la naturaleza y que la capacidad para proporcionar agua por parte de los sistemas ambientales es limitada.

En las intervenciones educativas de Havu-Nuutinen et al. (2011) y Havu-Nuutinen et al.(2017) en el marco de una instrucción CTS (Ciencia, Tecnología y Sociedad) sobre los problemas del agua, se analizaron las concepciones del alumnado y el proceso de cambio conceptual en escolares de primaria. Sus resultados manifiestan que, antes de la instrucción CTS, las concepciones de los escolares sobre el agua eran locales, descriptivas y subjetivas. Después del proceso de enseñanza, el uso de explicaciones científicas aumentó y adquirieron una concepción más global y objetiva del agua y establecieron conexiones entre los estados y la circulación del agua a través de los diferentes sistemas, e incluso fueron capaces de describir la conexión entre los recursos hídricos y la importancia del agua para la vida. 
La investigación de Fernández Arroyo (2012b) tiene como objetivo conocer los procesos de construcción del conocimiento en torno a la temática del agua en estudiantes de primero de bachillerato de un centro español. Las actividades programadas giran en torno a los temas ciclo del agua, la captación, distribución y potabilización de esta, así como los consumos del sector industrial, servicios y población, y las problemáticas ambiental y social sobre el agua y para analizar los resultados establecieron un sistema de categorías, desarrollando en cada una de ellas una hipótesis de transición. En la propuesta didáctica de Fernández-Arroyo y RodríguezMarín (2017) el objetivo era demostrar el potencial didáctico que la bipótesis de transición en contenidos como el uso, gestión y contaminación del agua, que tienen una alta carga conceptual, procedimental y actitudinal. La hipótesis de transición permite una organización de los contenidos que facilita la evolución del conocimiento del alumnado desde lo simple hacia lo complejo. De esta manera, respecto al ciclo del agua, el alumnado fue capaz de conectar el ciclo del agua a escala planetaria con el ciclo del agua a escala local. En referencia al ahorro del agua, el alumnado consiguió entender que la responsabilidad no recae solo en el sector población y que el manejo de los costes del uso y gestión del agua (económica, ambiental y social) son bastante complejos.

La intervención didáctica llevada a cabo por Mazas, Gil-Quílez, Martínez-Peña, Hervas y Muñoz (2018) tiene como objetivo mostrar la capacidad de modelización y del pensamiento sistemático en los niños de infantil, a través de una intervención didáctica sobre los cambios de estado. Tras la intervención, los escolares fueron capaces de construir un modelo sobre los cambios de estado, además de trabajar diversas destrezas científicas como la observación, la formulación de hipótesis, búsqueda de información, experimentación, la comunicación y/o discusión de resultados. Los autores destacan la importancia de iniciar a los escolares de la etapa infantil en la construcción de modelos y el desarrollo de destrezas científicas, y conectar este conocimiento con los que trabajarán en etapas posteriores de primaria y secundaria.

\section{Conclusiones}

El agua es y sigue siendo un contenido escolar presente en la investigación educativa. Dado el incremento cuantitativo de investigaciones que ha tenido lugar durante las dos últimas décadas, así como la mayor y más extendida aceptación de la necesaria educación ambiental de la ciudadanía, no resulta difícil afirmar que este número de investigaciones aumentará en la década que se inicia.

Por su carácter interdisciplinar y por la intensa relación entre sus contenidos, es difícil realizar una revisión bibliográfica con cierta sistematicidad. En este trabajo, se presenta una propuesta en la que la base de datos finalmente alcanzada está formada por 47 artículos distribuidos en 22 revistas del área de la educación científica. A pesar de las limitaciones, ya comentadas, de la metodología de búsqueda, estimamos que la información aportada por esta muestra de artículos es de alto interés para quienes pretenden indagar en cualquier temática relacionada con el agua.

Para el análisis de contenidos, se adoptó el criterio de analizar la relación existente entre el contenido investigado en el artículo y cada uno de los contenidos curriculares relacionados con el agua en la legislación española. Esta relación fue identificada mediante una cifra en una escala del uno al cuatro, en la que la importancia relativa del contenido curricular va disminuyendo progresivamente en el artículo de investigación. En este trabajo, únicamente se presentan los 47 artículos agrupados según el contenido principal. 
Sobre qué contenidos curriculares relacionados con el agua han sido más y menos investigados, que constituye la primera pregunta de investigación, este trabajo demuestra que el número de artículos centrado en la relación del agua con el medio físico es bastante superior al destinado a estudiar las relaciones del agua con los seres vivos. En el primer grupo se ubican los contenidos tradicionalmente incluidos en la física y en la química, y en el segundo, en el consumo y ahorro, en la gestión y en los problemas medioambientales. Ambos grupos de conocimientos no deberían ser tratados de forma independiente ni deberían verse como antagónicos, ni mucho menos, pero se hace necesario ahondar en las investigaciones relacionadas con el segundo grupo de trabajos para ayudar al alumnado a avanzar desde una concepción del agua local y subjetiva a otra más extensa en el espacio y en el tiempo, y más compartida, descentrada y objetiva. Se trata en definitiva de una perspectiva necesaria para comprender el rol proactivo que el propio estudiante ha de adoptar para transitar hacia la sostenibilidad de nuestro planeta.

El análisis de la distribución de los contenidos curriculares investigados a través de las etapas educativas ha mostrado que éstas son, en orden de más a menos frecuencia: primaria, secundaria obligatoria, bachillerato, infantil y universidad. No obstante, se ha de seguir indagando para confirmar este extremo. Asimismo, en trabajos anteriores ya se ha comprobado el olvido de la etapa de infantil en las investigaciones didácticas (Benarroch, 2010), posiblemente por las dificultades tanto teóricas como metodológicas que entraña este tipo de investigación y su alejamiento de la formación inicial de los investigadores.

En este mismo sentido, es evidente la importancia del ciclo del agua como pilar o contenido básico dentro del agua. Como ya destacó Massa en un trabajo pionero (1994) «son muchos y muy complejos los saberes que hay que manejar con respecto al agua y, entre todos ellos, el ciclo representa el alfabeto». Este carácter básico es probablemente el que hace que sea el contenido principal más abundante de todos los contenidos curriculares. No obstante, y ligando con el punto anterior, quizás haya que considerar, junto al ciclo natural del agua, los aspectos relacionados con el uso urbano y las cuestiones económicas y ambientales asociadas a la depuración, para que el alumnado llegue a comprender la extensión del problema de la escasez del agua en el planeta y la necesidad de una nueva, aunque ya no lo sea tanto, gestión y cultura del agua. De hecho, un conjunto de hidrólogos recientemente también se ha unido a la amplia denuncia que otros investigadores han realizado sobre los diagramas de los ciclos naturales del agua en los libros de texto, en este caso, para destacar que no se pueden dejar de lado las relaciones de los humanos con el agua de la Tierra para comprender los límites de la disponibilidad futura de este recurso esencial y escaso (Abbott et al. 2019).

En la segunda pregunta de investigación nos preguntábamos qué se sabe acerca de las dificultades de aprendizaje y de las estrategias de enseñanza de los contenidos relacionados con el agua. De los resultados expuestos en este trabajo, se pueden destacar las siguientes conclusiones:

- Las dificultades cognitivas son señaladas por distintos autores tanto en las primeras etapas de la enseñanza (Infantil, Primaria), para comprender por ejemplo la presencia de agua en el aire (Cruz-Guzmán et al. 2017), como en Secundaria, para dar visibilidad a las aguas subterráneas (Dickerson y Dawkins 2004). En esta misma línea, la ausencia del pensamiento sistémico (Ben-Zvi y Orion 2005b, 2010a) ha sido identificada como una causa de dificultades en la comprensión del ciclo del agua. 
- Las dificultades de aprendizaje de los estudiantes también se ven reforzadas por las imágenes de los libros de texto e ilustraciones de internet. El producto es una visión simple y errónea por ejemplo del ciclo del agua, limitado al flujo del agua sin vida, en el que no se alude en ocasiones a la existencia de las aguas subterráneas (Cardak 2009; Reyero et al. 2007; Hernández 2014) o solo se representan las cuencas en las áreas naturales y conectadas con los ríos (Shepardson et al. 2005). Los problemas con las aguas subterráneas permanecen incluso en el alumnado universitario (Fernández y González 2010; Romine et al. 2015). No obstante, el contacto con la naturaleza ayuda a comprender mejor y desde más jóvenes las relaciones del ser humano con el agua (Ben-Zvi et al. 2012; Endreny 2010) y la contaminación del agua Kiryak y Çalik 2018).

- Otro origen de las dificultades de los estudiantes parece estar en la cultura popular del agua y en el escaso tiempo que el profesorado dedica a erradicarla en las aulas (Jaén y Palop 2011).

- En cuanto a las estrategias de enseñanza, las estrategias por indagación contextualizadas en el movimiento CTS parecen ser útiles para avanzar en los conocimientos sobre cambios de estado en Infantil (Cruz-Guzmán et al. 2017), ciclo natural del agua (Ben-Zvi y Orion 2010b; Forbes et al. 2015; Zangori et al. 2015) el uso urbano del agua (Castelltort et al. 2014) en Primaria, así como también para avanzar en el conocimiento que, sobre las aguas subterráneas (Fernández y González 2013), sobre la contaminación (Cano 2007; Fernández y Solís 2011; Jaén et al. 2014) o sobre la depuración de las aguas residuales, tienen los estudiantes de Secundaria. Para los estudiantes de esta última etapa educativa, los modelos simulando acuíferos en distintas formas geológicas, pueden ayudar a mejorar las representaciones sobre las aguas subterráneas (Calvo et al. 2007) y sobre el ciclo del agua (Nebot 2007; Nebot y Márquez 2014; Otrel Cass 2017). Para Bratitsis et al. (2017) la realidad aumentada puede tener mucho futuro en la enseñanza del ciclo del agua en Primaria al ayudar a hacer visible lo invisible.

\section{Referencias bibliográficas}

Abbott B.W., Bishop K., Zarnetske J.P., Minaudo C., Chapin III F.S., Krause S., Hannah D.M., Conner L., Ellison D., Godsey S.E., Plont P., Marçais J., Kolbe T., Huebner A., Frei R.J., Hampton T., Gu S., Buhman M., Sayedi S.S., Ursache O.,...Pinay G. (2019) Human domination of the global water cycle absent from depictions and perceptions. Nature Geoscience 12, 533-540. https://doi.org/10.1038/s41561-019-0374-y.

Agraso M.F., Jiménez M.P. (2003) Percepción de los problemas ambientales por el alumnado: los recursos naturales. Didáctica de las Ciencias Experimentales y Sociales 17, 91-105.

Ahi B. (2017) The effect of talking drawings on five-year-old turkish children's mental models of the water cycle. International Journal of Environmental and Science Education 12(3), 349367. https://doi.org/10.12973/ijese.2017.01232a.

Benarroch A. (2010) La investigación en Didáctica de las Ciencias Experimentales en las etapas educativas de Infantil y Primaria. En A.M. Abril y A. Quesada (eds.) Actas de los XXIV Encuentro de Didáctica de las Ciencias Experimentales, 32-52.

Ben-Zvi Assaraf O., Orion N. (2005a) Development of system thinking skills in the context of Earth System education. Journal of Research in Science Teaching 42(5), 518-560. https://doi.org/10.1002/tea.20061. 
Ben-Zvi Assaraf O., Orion N. (2005b) A study of junior high students' perceptions of the water cycle. Journal of Geoscience Education 53(4), 366-373. https://doi.org/10.5408/1089-9995-53.4.366.

Ben-Zvi Assaraf O., Orion N. (2010a) Four case studies, six years later: Developing system thinking skills in junior high school and sustaining them over time. Journal of Research in Science Teaching 47(10), 1253-1280. https:/ /doi.org/10.1002/tea.20383.

Ben-Zvi Assaraf O., Orion N. (2010b) System thinking skills at the elementary school level. Journal of Research in Science Teaching 47(5), 540-563. https://doi.org/10.1002/tea.20351.

Ben-Zvi Assaraf O., Eshach H., Orion N., Alamour Y. (2012) Cultural differences and students' spontaneous models of the water cycle: A case study of jewish and bedouin children in Israel. Cultural Studies of Science Education 7, 451-477. https://doi.org/10.1007/s11422-012-9391-5.

Bratitsis T., Bardanika P., Ioannou M. (2017) Science education and augmented reality content: The case of the water circle. 17th International Conference on Advanced Learning Technologies. In 2017 IEEE, 485-489. https:// doi.org/10.1109/ICALT.2017.64.

Cano M.I. (2007) La contaminación del agua: una propuesta para trabajar de forma funcional y significativa en la educación secundaria. Investigación en la Escuela 63, 47-63.

Cardak O. (2009) Science students' misconceptions of the water cycle according to their drawings. Journal of Applied Sciences 9(5), 865-873. https://doi.org/10.3923/jas.2009.865.873.

Castelltor A. (2015) Actividades que contribuyen a la promoción de una nueva cultura ambiental del agua. Comunicações 22(2), 363-389. https://doi.org/10.15600/2238121X/comunicacoes.v22n2ep363-389.

Castelltort A., Sanmartí N., Pujol D. (2014) Actividades en el entorno: una oportunidad para aprender sobre el agua. Alambique. Didáctica de las Ciencias Experimentales 77, 54-61.

Calvo M., Reyero C., Vidal M.P., Morcillo J.G., García E. (2007) El trabajo con modelos en aguas subterráneas. Enseñanza de las Ciencias de la Tierra 15(3), 341-347.

Covitt B.A., Gunckel K.L., Anderson C.W. (2009) Students' developing understanding of water in environmental systems. The Journal of Environmental Education 40(3), 37-51. https://doi.org/10.3200/JOEE.40.3.37-51.

Cruz-Guzmán M., García-Carmona A., Criado A.M. (2017) Aprendiendo sobre los cambios de estado en educación infantil mediante secuencias de pregunta-prediccióncomprobación experimental. Enseñanza de las Ciencias 35(3), 175-193. https://doi.org/10.5565/rev/ensciencias.2336.

Çoban G.Ü., Akpinar E., Küçükcankurtaran E., Yildiz E., Ergin Ö. (2011) Elementary school students' water awareness. International Research in Geographical and Environmental Education 20(1), 65-83. https://doi.org/10.1080/10382046.2011.540103.

Dickerson D., Dawkins K. (2004) Eighth grade students' understandings of groundwater. Journal of Geoscience Education 52, 178-181. https://doi.org/10.5408/1089-9995-52.2.178

Endreny A.H. (2010) Urban 5th graders conceptions during a place-based inquiry unit on watersheds. Journal of Research in Science Teaching 47(5), 501-517. https://doi.org/10.1002/tea.20348. 
Fernández G., González F. (2010) El problema de la descarga del agua subterránea al medio superficial: estudio de esquemas de conocimiento en universitarios. Didáctica de las Ciencias Experimentales y Sociales 24, 153-169.

Fernández G., González F. (2013) Propuesta didáctica basada en la investigación dirigida: la importancia del agua subterránea en el caudal de los ríos. Enseñanza de las Ciencias de la Tierra 21(1), 84-90.

Fernández J., Solís E. (2011) El agua como recurso para investigar en el aula. Una investigación en la asignatura de Ciencias para el Mundo Contemporáneo. Investigación en la Escuela $75,49-61$.

Fernández Arroyo J. (2012a) La construcción del conocimiento sobre la gestión y la contaminación del agua. Concepciones del alumnado de $1^{\circ}$ de Bachillerato. Tesis doctoral. Sevilla. Fondo digital de la Universidad de Sevilla.

Fernández Arroyo J. (2012b) Los procesos de construcción del conocimiento significativo del agua en bachillerato. Estudio de casos. Enseñanza de las Ciencias 30(3), 177-194. https://doi.org/10.5565/rev/ec/v30n3.561.

Fernández-Arroyo J., Rodríguez-Marín F. (2017) Los procesos de enseñanza-aprendizaje relacionados con el agua en el marco de la hipótesis de transición. Revista Eureka Sobre Enseñanza y Divulgación de las Ciencias 14(1), 227-243. https://doi.org/10.25267/Rev_Eureka_ensen_divulg_cienc.2017.v14.i1.17.

Forbes C.T., Zangori L., Schwarz C.V. (2015) Empirical validation of integrated learning performances for hydrologic phenomena: 3rd-grade students' model-driven explanation-construction. Journal of Research in Science Teaching 52(7), 895-921. https://doi.org/10.1002/tea.21226.

Gunckel K.L., Covitt B.A., Salinas I., Anderson, C.W. (2012) A learning progression for water in socio-ecological systems. Journal of Research in Science Teaching 49(7), 843-868. https://doi.org/10.1002/tea.21024.

Havu-Nuutinen S., Kärkkäinen S., Keinonen T. (2011) Primary school pupils' perceptions of water in the context of STS study approach. International Journal of Environmental and Science Education 6(4), 321-339.

Havu-Nuutinen S., Kärkkäinen S., Keinonen T. (2017) Changes in primary school pupils' conceptions of water in the context of Science, Technology, and Society (STS) instruction. International Research in Geographical and Environmental Education 27(2), 118134. https://doi.org/10.1080/10382046.2017.1320897.

Henriques L. (2002) Children's ideas about weather: a review of the literature. School Science and Mathematics 102(5), 202-215. https://doi.org/10.1111/j.1949-8594.2002.tb18143.x.

Hernández M.J. (2014) ¿Qué debería conocer todo ciudadano sobre el agua? Alambique. Didáctica de las Ciencias Experimentales 77, 9-16.

Jaén M., Esteve P., de Pro Bueno A. (2014) ¿Ingenio o ingeniería? Actividades sobre problemas de contaminación de las aguas. Alambique. Didáctica de las Ciencias Experimentales 77, 35-44.

Jaén M., Palop E. (2011) ¿Qué piensan y cómo actúan los alumnos y profesores de un Centro de Educación Secundaria sobre la gestión del agua, la energía y los residuos? Enseñanz̧a de las Ciencias 29(1), 61-74. 
Kiryak Z., Çalik M. (2018) Improving grade 7 students' conceptual understanding of water pollution via common knowledge construction model. International Journal of Science and Mathematics Education 16, 1025-1046. https://doi.org/10.1007/s10763-017-9820-8.

Köseoğlu P., Soran H., Storer J. (2009) Developing learning stations for the purification of waste water. World Conference on Educational Sciences. In Procedia Social and Behavioral Sciences 1, 210-214. https://doi.org/10.1016/j.sbspro.2009.01.038.

Malleus E., Kikas E., Kruus S. (2016) Students' understanding of cloud and rainbow formation and teachers' awareness of students' performance. International Journal of Science Education 38(6), 993-1011. https://doi.org/10.1080/09500693.2016.1175683.

Malleus E., Kikas E., Marken T. (2017) Kindergarten and primary school children's everyday, synthetic, and scientific concepts of clouds and rainfall. Research in Science Education 47, 539-558. https://doi.org/10.1007/s11165-016-9516-z.

Marcén C. (2006) El aprendizaje de las ideas de los escolares sobre el agua no surge porque sí. En La Educación Ambiental en Aragón en los albores del siglo XXI (pp 130-135). Zaragoza: Gobierno de Aragón, Departamento de Desarrollo Rural y Sostenibilidad, España.

Márquez C., Bach J. (2007) Una propuesta de análisis de las representaciones de los alumnos sobre el ciclo del agua. Enseñanza de las Ciencias de la Tierra 15(3), 280-286.

Massa B. (1994) Alphabétisation en science de la terre: conceptions et obstacles cognitifs sur le cycle de l'eau à l'école primaire. En Giordan A.; Martinand J.L. (Eds). Actes JIES XVI, 313-318.

Mazas B., Gil-Quílez M.J., Martínez-Peña B., Hervas A., Muñoz A. (2018) Los niños y las niñas de infantil piensan, actúan y hablan sobre el comportamiento del aire y del agua. Enseñanza de las Ciencias 36(1), 163-180. https://doi.org/10.5565/rev/ensciencias.2320.

MEC (2007) Real Decreto 1630/2006, de 29 de diciembre, por el que se establecen las enseñanzas mínimas del segundo ciclo de Educación infantil. BOE núm. 4, de 4 de enero de 2007, pp. 474-482.

MEC (2014) Real Decreto 126/2014, de 28 de febrero, por el que se establece el currículo básico de la Educación Primaria. BOE núm. 52, de 1 de marzo de 2014, pp. 1-58.

MEC (2015) Real Decreto 1105/2014, 26 de diciembre, por el que se establece el currículo básico de la Educación Secundaria Obligatoria y del Bachillerato. BOE núm. 3, de 3 de enero de 2015, pp. 169-546.

Miller E., Manz E., Russ R., Stroupe D., Berland L. (2018) Addressing the epistemic elephant in the room: Epistemic agency and the next generation science standards. Journal of Research in Science Teaching 55(7). https://doi.org/1053-1075. 10.1002/tea.21459.

Nebot M.R. (2007) El ciclo del agua en una garrafa. Enseñanza de las Ciencias de la Tierra 15(3), 333-340.

Nebot M.R., Márquez C. (2014) El ciclo del agua en el laboratorio. Alambique. Didáctica de las Ciencias Experimentales 77, 17-24.

Otrel-Cass K. (2017) Reflective reconstruction of visual products: Studying the water cycle. Knowledge Cultures 5(5), 14-28. https://doi.org/10.22381/KC5520172.

Pascual J.A. (2014) Conflictos socioambientales en torno al agua. Alambique. Didáctica de las Ciencias Experimentales 77, 25-34. 
Reinfried S., Aeschbacher U., Kienzler P.M., Tempelmann S. (2015) The model of educational reconstruction - A powerful strategy to teach for conceptual development in physical geography: The case of water springs. International Research in Geographical and Environmental Education 24(3), 237-257. https://doi.org/10.1080/10382046.2015.1034459.

Reyero C., Calvo M., Vidal M.P., García E., Morcillo J.G. (2007) Las ilustraciones del ciclo del agua en los textos de Educación Primaria. Enseñanza de las Ciencias de la Tierra 15(3) 287294.

Roca M., Márquez C., Sanmartí N. (2013) Las preguntas de los alumnos: Una propuesta de análisis. Enseñanza de las Ciencias 31(1), 95-114. https://doi.org/10.5565/rev/ec/v31n1.603.

Romine W.L., Schaffer D.L., Barrow L. (2015) Development and application of a novel raschbased methodology for evaluating multi-tiered assessment instruments: Validation and utilization of an undergraduate diagnostic test of the water cycle. International Journal of Science Education 37(16), 2740-2768. https://doi.org/10.1080/09500693.2015.1105398.

Sammel A., McMartin D., Arbuthnott K. (2018) Education agendas and resistance with the teaching and learning of freshwater and extreme freshwater events. Australian Journal of Environmental Education 34(1), 18-32. https://doi.org/10.1017/aee.2018.10.

Shepardson D.P., Harbor J., Wee B. (2005) Water towers, pump houses, and mountain streams: Students' ideas about watersheds. Journal of Geoscience Education 53(4), 381-386. https://doi.org/10.5408/1089-9995-53.4.381.

Shepardson D.P., Wee B., Priddy M., Schellenberger L., Harbor J. (2009) Water transformation and storage in the mountains and at the coast: Midwest students' disconnected conceptions of the hydrologic cycle. International Journal of Science Education 31(11), 1447-1471. https://doi.org/10.1080/09500690802061709.

Zangori L., Forbes C.T., Schwarz C.V. (2015) Exploring the effect of embedded scaffolding within curricular tasks on third-grade students'model-based explanations about hydrologic cycling. Science and Education 24, 957-981. https://doi.org/10.1007/s11191015-9771-9. 